\title{
Avaliação genética de características de escores visuais de bovinos da raça Nelore da desmama até a maturidade ${ }^{1}$
}

\section{Carina Ubirajara de Faria², Cláudio de Ulhôa Magnabosco ${ }^{3}$, Lucia Galvão de Albuquerque 4 , Luiz Antônio Framartino Bezerra ${ }^{5}$, Raysildo Barbosa Lôbo ${ }^{5}$}

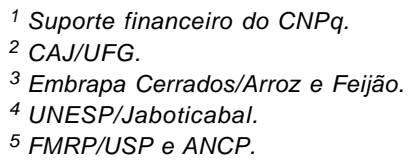

RESUMO - Os objetivos neste estudo foram estimar parâmetros genéticos das características categóricas musculosidade, estrutura física, aspectos raciais, conformação, ônfalo, pigmentação e sacro e predizer os valores genéticos utilizando-se a estatística bayesiana sob modelo animal de limiar, considerando diferentes idades de bovinos da raça Nelore. As informações de escores visuais foram obtidas nos anos de 2000 a 2005 de bovinos provenientes de 13 fazendas participantes do Programa Nelore Brasil. Nas análises bicaracterísticas, foram utilizados 500.000 até 1.100.000 ciclos para alcançar a convergência da cadeia de Gibbs. O descarte inicial e o intervalo amostral foram de 100.000 e 1.000 ciclos, respectivamente. As características de escores visuais avaliadas aos 8 e 22 meses de idade apresentaram estimativas de herdabilidades moderadas, indicando resposta rápida à seleção direta. Os escores visuais indicaram possibilidade de resposta rápida à seleção direta e, portanto, devem ser incorporados em programas de melhoramento genético como critérios de seleção. As estimativas de correlações genéticas entre musculosidade, estrutura física e conformação também indicam que a seleção direta para uma destas características trará progresso genético às outras. Recomenda-se utilizar os escores visuais como critérios de seleção em pelo menos duas fases de vida do animal, na desmama e ao sobreano.

Palavras-chave: amostragem de Gibbs, características morfológicas, parâmetros genéticos, Zebu

\section{Genetic evaluation of visual scores traits of Nelore cattle from weaning to maturity}

\begin{abstract}
The objectives of this study were toestimate the genetic parameters of the following categorical traits: musculature, physical structure, racial breed aspects, conformation, navel, pigmentation and sacrum, and predict breeding values, using bayesian statistics under threshold animal models, considering different ages of cattle of the Nelore breed. The information concerning visual scores were obtained from 2000 to 2005 from cattle of the Nelore breed from thirteen participant farms of the Brazilian Nelore Program. In the two-trait analyses, 500,000 rounds up to 1,100,000 cycles were used to reach the convergence of the Gibbs chain. The burn-in and thinning interval were 100,000 and 1,000 cycles, respectively. The evaluated visual scores at the age of 8 and 22 months presented moderate heritability estimates, suggesting that these traits can be used to promote genetic gain by direct selection. The genetic correlations between the morphological traits of musculature, physical structure and conformation were moderate, indicating correlated responses between the traits. The use of the visual scores is recommended, as selection criteria, in at least two phases of the animal life, at weaning and yearling time.
\end{abstract}

Key Words: genetic parameters, Gibbs sampling, morphological traits, zebu cattle

\section{Introdução}

A seleção de animais por um biótipo adequado ao sistema produtivo é um aspecto determinante para aumentar a eficiência econômica da empresa rural e condição primordial para a sustentabilidade da atividade pecuária. Nesse sentido, escores de avaliação visual têm sido inclu ídos em programas de melhoramento genético para identificar indivíduos com melhor carcaça e que chegarão com maior rapidez ao abate.

Com o objetivo de encontrar animais que reúnam maior número de características de importância econômica e de 
melhorar alguns aspectos relacionados ao biótipo animal, no Programa Nelore Brasil da Associação Nacional de Criadores e Pesquisadores (ANCP), foi desenvolvido o método de avaliação visual por escores denominado MERCOS (Lôbo, 1996), em que são consideradas as características morfológicas de musculosidade, estrutura física, aspectos raciais, conformação, ônfalo e aspectos sexuais.

No entanto, na avaliação genética de características morfológicas, em que os dados não apresentam distribuição normal, alguns cuidados devem ser considerados, uma vez que as metodologias de modelos lineares geralmente resultam em baixas estimativas de herdabilidade (Mwansa et al., 2002). Assim, recomenda-se o uso de modelos de limiar, que apresentam maior habilidade para detecção da variabilidade genética em comparação aos modelos lineares (Ramirez Valverde et al., 2001; Luo et al., 2002; Marcondes et al., 2005).

O modelo de limiar aplicado em análises genéticas de variáveis discretas, distribuídas em classes, se baseia na suposição de que as classes de dados observáveis estão relacionadas a um delineamento de uma variável normal ou escala subjacente contínua (Sorensen et al., 1995; Van Tassel et al., 1998). Dessa maneira, assume-se que existe uma variável aleatória não-observável, associada aos níveis de cada característica categórica, contendo os efeitos fixos e aleatórios.

Os objetivos neste trabalho foram estimar os parâmetros genéticos das características de escores visuais e predizer os valores genéticos utilizando-se a estatística bayesiana sob modelo animal de limiar, considerando as idades de bovinos da raça Nelore. A obtenção das estimativas de herdabilidade poderá contribuir para a elaboração e implantação de programas de melhoramento genético e para determinação da fase de vida do animal que proporciona melhor resposta à seleção.

\section{Material e Métodos}

As informações de escores visuais analisadas neste estudo foram obtidas entre os anos de 2000 e 2005, em bovinos da raça Nelore, provenientes de 13 fazendas participantes do Programa Nelore Brasil, da Associação Nacional de Criadores e Pesquisadores (ANCP), localizadas nos estados de Goiás, Mato Grosso, Paraná e no Distrito Federal. Foram estudadas as características morfológicas de musculosidade, estrutura física, aspectos raciais, conformação, ônfalo e sacro.

Os animais foram avaliados visualmente pelo método denominado MERCOS, adaptado de Lôbo (1996), em que: para musculosidade, avaliam-se a distribuição muscular no corpo do animal e seu desenvolvimento, volume e comprimento dos músculos; para estrutura física, avalia-se a sustentação do animal, ou seja, os cascos, quanto à integridade e ao tamanho, ligamentos ou articulações quanto à firmeza e à largura dos ossos; para os aspectos raciais, avalia-se o padrão racial identificando os animais que se adequam melhor ao perfil da raça considerando os defeitos como desvios de chanfro e articulação dos maxilares; para a conformação, avaliam-se o esqueleto do animal, o comprimento do corpo, a amplitude torácica, o arqueamento e o comprimento das costelas, a largura de garupa e o comprimento, a relação tamanho dos membros e o comprimento das costelas, observando-se que as linhas dorsal e ventral devem ser paralelas ou abrir levemente no sentido da garupa; no ônfalo, é avaliado o umbigo, que deve apresentar tamanho e posicionamento adequados, tomando-se como base animais de umbigo com tamanho reduzido. Neste estudo, os aspectos sexuais não foram considerados. De acordo com Lôbo (1996), para cada variável morfológica, os animais avaliados podem apresentar escores de 1 a 5 pontos, de modo que a maior pontuação representa o grau mais favorável.

Além das características morfológicas do sistema de avaliação visual MERCOS, foi avaliado também o sacro. Para atribuir escores para sacro, avaliaram-se o comprimento, por meio da distância entre os ossos íleo e ísquio, a largura, considerando a distância entre os íleos e entre os ísquios, e a inclinação do osso sacro. Como sacro é uma característica binária ou dicotômica, foram utilizados apenas dois escores, assumindo-se o valor 1 para animais com osso sacro indesejável e valor 2 para osso sacro desejável.

Para a avaliação do ônfalo, a escala de notas variou de acordo com uma referência previamente definida, de modo que o escore ideal ( 5 pontos) foi relativo aos animais com umbigo de tamanho reduzido e posicionamento adequado. $\mathrm{Na}$ avaliação de vacas adultas, os escores atribuídos para todas as características morfológicas foram relativos a um biótipo de referência da raça, não sendo utilizado o critério comparativo dentro de lote de manejo (Tabela 1).

Em cada lote de manejo, os animais foram analisados individualmente pelo mesmo avaliador, ou seja, avaliaramse animais de mesmo sexo nascidos na mesma época e que receberam as mesmas condições de tratamento alimentar e sanitário. Primeiramente, observou-se todo o lote de manejo a fim de visualizar o perfil médio para cada característica morfológica avaliada. Desta forma, a avaliação visual foi comparativa e a pontuação dada para cada indivíduo relativa aos demais.

Para a consistência dos dados, os intervalos de idade dos animais jovens utilizados neste estudo variaram de 
Tabela 1 - Distribuição (\%) por escores para as características musculosidade, estrutura física, aspectos raciais, conformação, ônfalo e sacro de bovinos da raça Nelore considerandodiferentesidades

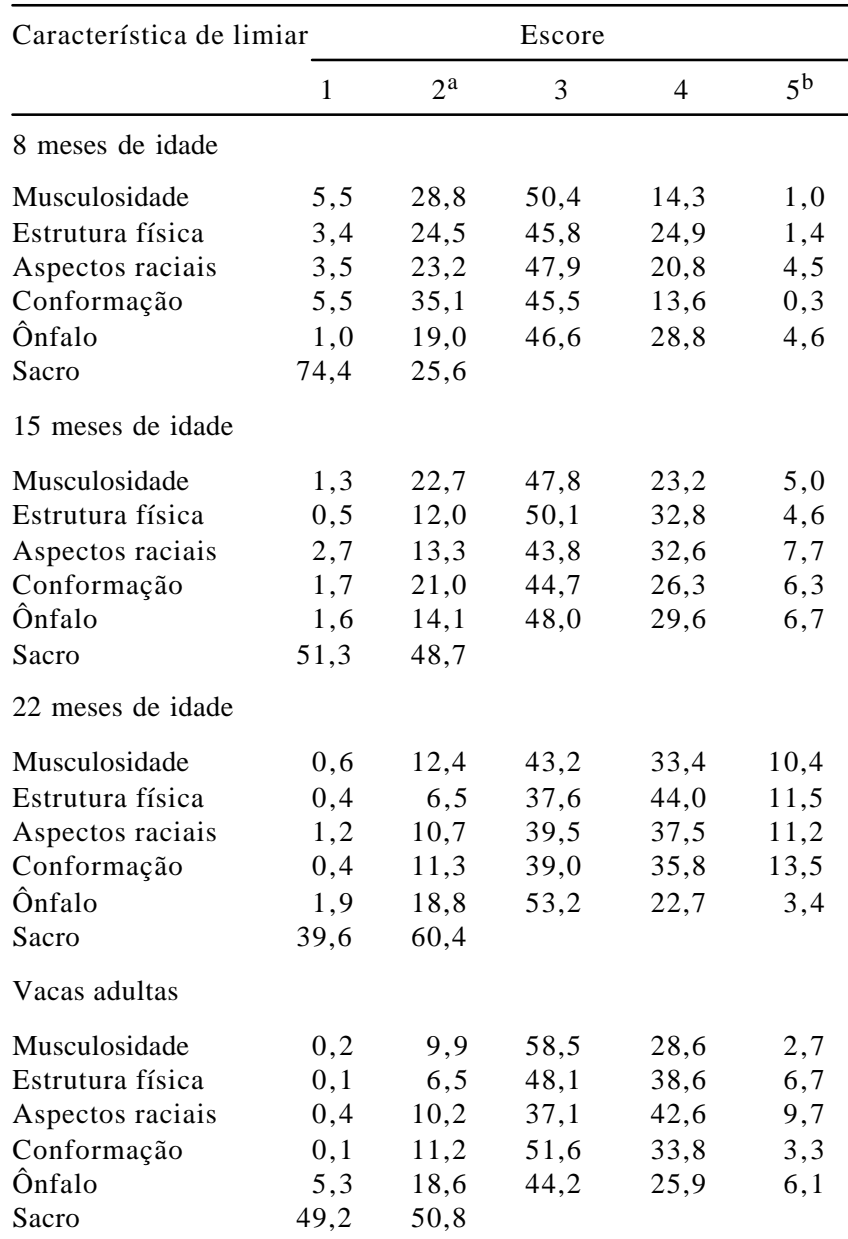

${ }^{\mathrm{a}}$ Escore ideal para sacro; ${ }^{\mathrm{b}}$ Escore ideal para musculosidade, estrutura física, aspectos raciais, conformação e ônfalo.

150 a 330 dias em relação aos 240 dias (8 meses de idade), 360 a 540 dias em relação aos 450 dias ( 15 meses de idade) e 570 a 750 dias em relação aos 660 dias (22 meses de idade), considerando um período de 90 dias para menos e para mais em cada classe de idade pré-definida. Para as vacas adultas, a variação da idade foi de 4 a 12 anos, conforme critério adotado pelo Programa Nelore Brasil para o peso adulto da vaca (Lôbo et al., 2006). Na verificação dos efeitos de ambiente que influenciam as características morfológicas, foram utilizados os procedimentos GLM e REG (SAS, 2004).

A definição dos grupos de contemporâneos foi determinada por: i) fazenda, ano e estação de nascimento, regime alimentar e lote de manejo aos 210 dias para os animais de 8 meses de idade; ii) fazenda, ano e estação de nascimento, lote de coleta para animais de 15 e 22 meses de idade; iii) fazenda, ano e estação de nascimento para vacas adultas. No entanto, para o peso aos 210 dias de idade, os grupos contemporâneos incluíram animais da mesma fazenda, ano e estação de nascimento, sexo e lote de manejo aos 210 dias de idade. O efeito de estação de nascimento foi dividido em quatro classes: animais nascidos nos meses de janeiro a março, abril a junho, julho a setembro e outubro a dezembro. Foram eliminados os grupos contemporâneos com menos de quatro animais para todas as características avaliadas. Para o peso aos 210 dias de idade, além desta restrição, foram retirados os animais com medidas de 3,5 desvios-padrão acima ou abaixo da média do seu grupo de contemporâneos.

Os parâmetros genéticos das características morfológicas para as diferentes idades foram estimados mediante análises bicaracterísticas sob modelo animal linear-limiar utilizando-se a estatística bayesiana por meio do aplicativo MTGSAM Threshold (Multiple Trait Gibbs Sampler for Animal Models) desenvolvido por Van Tassel et al. (1998). O modelo completo pode ser representado em notação matricial como :

$$
y=X \beta+Z_{1} a+Z_{2} m+Z_{3} c+e
$$

em que: $y=$ vetor das observações (características morfológicas e peso aos 210 dias de idade); $\beta=$ vetor dos efeitos fixos (sexo, classe de idade da vaca ao parto ou ano e época da coleta para escores visuais e grupo contemporâneo para P210); $a$ = vetor dos efeitos aleatórios que representam os valores genéticos aditivos diretos de cada animal; $m=$ vetor dos efeitos aleatórios que representam os valores genéticos aditivos maternos (somente para características morfológicas avaliadas à desmama); $c=$ vetor de efeitos aleatórios não correlacionados (efeito de grupos contemporâneos para as características morfológicas ou efeito de ambiente permanente materno para peso aos 210 dias); $e=$ vetor de efeitos aleatórios residuais; e $X, Z_{1}$, $Z_{2}$ e $Z_{3}=$ matrizes de incidência que relacionam as observações aos efeitos fixos e aos efeitos aleatórios genéticos aditivos direto e maternal, e não correlacionados, respectivamente. $\mathrm{O}$ arquivo de genealogia incluiu 52.361 animais da raça Nelore.

Para a característica contínua de peso aos 210 dias de idade, não foi incluído o efeito genético aditivo materno, em virtude da dificuldade de se estimar todas as variâncias e covariâncias genéticas sob o modelo de limiar. No entanto, modelos que não incluem os efeitos maternos podem inflacionar a variância genética aditiva direta e levar a estimativas mais altas da herdabilidade (Meyer, 1992). Para evitar superestimação da variância genética aditiva direta decorrente da não-inclusão do efeito genético aditivo materno, foi incluído o efeito não-correlacionado de ambiente permanente materno. No arquivo de dados, apenas 
$20 \%$ das mães possuíam somente um filho, o que permitiu a inclusão desse efeito no modelo.

Observou-se maior número de dados para animais com idades mais avançadas (Tabela 2), uma vez que as amostras de animais avaliados visualmente por escores foram diferentes para cada faixa etária.

Os modelos de limiar geralmente apresentam problemas na estimação de componentes de variância e predição dos valores genéticos quando se tem grande número de níveis de efeitos fixos (Moreno et al., 1997; Varona et al., 1999; Luo et al., 2002). Desta maneira, os efeitos de grupos de contemporâneos foram assumidos como aleatórios, e os efeitos de sexo, classe de idade da vaca ao parto ou ano e estação de coleta, como fixos para as análises genéticas de características categóricas.

No modelo de limiar, assume-se que a escala subjacente apresenta distribuição normal contínua, representada como:

$$
\mathrm{U} \mid \theta \sim \mathrm{N}\left(\mathrm{W} \theta, \mathrm{I} \sigma_{\mathrm{e}}^{2}\right)
$$

em que $U=$ vetor da escala base de ordem $r ; \theta^{\prime}=\left(\beta^{\prime}, a^{\prime}\right.$, $\left.m^{\prime}, c^{\prime}\right)=$ vetor dos parâmetros de locação de ordem $s$ com $\beta$ (definido sob o ponto de vista frequentista, como efeitos fixos) e ordem $s$ com $a, m$ e $c$ (como efeitos aleatórios genético aditivo direto, maternal e de grupo de contemporâneos); $W=$ matriz de incidência conhecida de ordem $r$ por $s ; I=$ matriz de identidade de ordem $r$ por $r$; e $\sigma^{2}{ }_{\mathrm{e}}=$ variância residual. Uma vez que a variável na distribuição subjacente não é observável, a parametrização

Tabela 2 - Descrição dos arquivos de dados analisados para as características musculosidade, estrutura física, aspectos raciais, conformação, ônfalo e sacro de bovinos da raça Nelore em diferentes idades

\begin{tabular}{lcccc}
\hline Escore visual & \multicolumn{4}{c}{ Número } \\
\cline { 2 - 5 } & Animais & Touros & Vacas & GC \\
\hline 8 meses de idade & & 108 & 952 & 30 \\
MERCO & 1.041 & 102 & 839 & 26 \\
SAC & 909 & & & \\
15 meses de idade & & & & \\
MERCO & 1.894 & 216 & 1.749 & 75 \\
SAC & 1.666 & 191 & 1.566 & 67 \\
22 meses de idade & & & & \\
MERCO & 3.864 & 301 & 3.093 & 118 \\
SAC & 3.434 & 261 & 2.776 & 102 \\
Vacas adultas & & & & \\
MERCO & 3.156 & 332 & 2.689 & 131 \\
SAC & 2.697 & 277 & 2.291 & 91 \\
\hline
\end{tabular}

GC: grupo contemporâneo. $\sigma^{2}{ }_{\mathrm{e}}=1$ é, geralmente, adotada no sentido de se obter identificabilidade na função de verossimilhança (Gianola \& Sorensen, 2002). Essa pressuposição é padrão em análises de dados categóricos sob modelo de limiar.

As características categóricas são determinadas por variáveis contínuas não-observáveis, em escala subjacente, por meio da fixação de valores iniciais de limiares, em que $t_{1}<t_{2} \ldots<t_{j-1}$, com $t_{0}=-\infty$ e $t_{j}=\infty$, de modo que $j$ é o número de categorias. Os dados observáveis são dependentes da variável subjacente, que é limitada entre dois limiares não-observáveis (Gianola \& Foulley, 1983). Assim, as categorias ou os escores de $y_{i}$ (características morfológicas) para cada animal $i$ são definidos por $U_{i}$ na escala s ubjacente:

$$
y_{i}= \begin{cases}1 & t_{0}<U_{i} \leq t_{1} \\ 2 & t_{1}<U_{i} \leq t_{2} \\ 3 & t_{2}<U_{i} \leq t_{3} \\ 4 & t_{3}<U_{i} \leq t_{4} \\ 5 & t_{4}<U_{i} \leq t_{5} \quad i=1, \ldots, n\end{cases}
$$

em que: $n=$ número de observações. Após as especificações dos limiares $t_{0}$ a $t_{5}$, é necessário que um dos limiares (de $t_{1}$ a $t_{4}$ ) seja ajustado a uma constante arbitrária. Neste estudo, assumiu-se $t_{1}=0$, de modo que o vetor de limiares estimáveis foi definido como:

$$
t=\left[\begin{array}{l}
t_{2} \\
t_{3} \\
t_{4}
\end{array}\right]
$$

Nas análises bicaracterísticas envolvendo variáveis categóricas e contínuas, de acordo com o enfoque bayesiano, admitiu-se que as distribuições iniciais dos efeitos aleatórios genéticos, não correlacionados e residuais, seguem distribuição normal multivariada conforme representado a seguir:

$$
p\left(\left[\begin{array}{l}
a_{1} \\
a_{2} \\
m_{1}
\end{array}\right] G\right) \sim \mathrm{N}\left(\left[\begin{array}{l}
0 \\
0 \\
0
\end{array}\right], \mathrm{G}=G_{0} \otimes A\right)
$$

$$
\begin{aligned}
& p\left(\begin{array}{l}
c_{1} \\
c_{2}
\end{array} \mid C\right) \sim \mathrm{N}\left(\left[\begin{array}{l}
0 \\
0
\end{array}\right], \mathrm{C}=C_{0} \otimes I\right) \\
& p\left(\begin{array}{l}
e_{1} \\
e_{2}
\end{array} \mid R\right) \sim \mathrm{N}\left(\left[\begin{array}{l}
0 \\
0
\end{array}\right], \mathrm{R}=R_{0} \otimes I\right)
\end{aligned}
$$

em que $G_{0}=$ matriz de variâncias e covariâncias genéticas; $C_{0}=$ matriz das variâncias de efeito de ambiente permanente 
materno (P210) e de grupo de contemporâneos (características morfológicas); $R_{0}=$ matriz de variâncias residuais; $\otimes=$ operador do produto direto; $A=$ matriz de parentesco; e $I=$ matriz identidade. Assumiram-se distribuições iniciais das covariâncias como Wishart Invertida para os efeitos aleatórios genéticos, não correlacionados e residuais das características estudadas, inclusive a covariância entre elas. Foram definidas distribuições iniciais uniformes, tanto para os efeitos fixos como para os limiares.

O parâmetro $v$ é o grau de liberdade correspondente à distribuição Wishart Invertida e indica o grau de confiabilidade da distribuição inicial. Neste estudo, o parâmetro $v$ foi considerado zero, ou seja, não refletia grau de conhecimento sobre os parâmetros.

Na implementação da Amostragem de Gibbs, utilizou-se cadeia inicial de 500.000 ciclos, de modo que os primeiros 100.000 ciclos foram descartados e as amostras retiradas a cada 1.000 ciclos, totalizando 400 amostras iniciais. Em seguida, foi dada continuidade à análise, adicionando 100.000 ciclos a cada rodada até que as estimativas obtidas na última análise fossem iguais às obtidas na análise anterior. Esse critério foi utilizado no intuito de verificar se realmente a convergência foi alcançada. As análises das amostras, da correlação serial e da convergência da cadeia de Gibbs foram realizadas com o auxílio do programa GIBANAL (Van Kaam, 1998).

Para obter as correlações genéticas entre os escores visuais, considerando as diferentes idades, foram realizadas análises bicaracterísticas sob modelo animal de limiar-limiar. Nestas análises, outra parametrização foi adotada (Van Tassel et al., 1998) e a variância residual foi estimável para as características morfológicas, bem como a covariância residual entre elas, e o $t_{1}$ igual a zero e o $t_{2}$ igual a um.

O erro de Monte Carlo foi estimado calculando a variância das amostras retiradas para cada componente de covariância e dividindo esta variância pelo número de amostras.

\section{Resultados e Discussão}

Para as análises bicaracterísticas, foram utilizados 500.000 até 1.100 .000 ciclos, de acordo com a necessidad e de cada análise para alcançar a convergência da cadeia de Gibbs.

A média, moda e mediana das estimativas de herdabilidade foram similares para todas as características morfológicas avaliadas em diferentes idades, exceto para o ônfalo aos 8 meses de idade, em que a moda foi maior que a média e a mediana (Tabela 3). Esses resultados indicam que a convergência da cadeia de Gibbs foi atingida e as distribuições marginais posteriores dos componentes de covariâncias tenderam a distribuição normal. Ressalta-se que a simetria das estimativas de medidas de tendência central é um indicativo da convergência da cadeia amostral e de uma análise acurada (Silva et al., 2005). No entanto, não é necessário que as estimativas das medidas de tendência central dos componentes de covariância sejam similares, pois esperam-se distribuições Wishart Invertida das densidades marginais posteriores dos componentes de covariâncias (Sorensen et al., 1995; Van Tassel et al., 1998).

O erro de Monte Carlo foi muito pequeno para todas as estimativas de herdabilidade (Tabela 3 ) e indica que o tamanho da cadeia de Gibbs foi suficiente para obter estimativas precisas das médias posteriores. O erro de Monte Carlo é considerado pequeno quando seu valor somado à estimativa média da distribuição posterior dos coeficientes de herdabilidade não altera o valor desta estimativa, considerando até a segunda casa decimal da herdabilidade. Assim, pode-se inferir que a metodologia utilizada foi eficiente para obter estimativas de parâmetros genéticos para características morfológicas categóricas.

As estimativas de herdabilidade direta obtidas para as características de musculosidade, estrutura física, conformação e ônfalo, avaliadas ao desmame, foram de magnitudes moderadas a altas (Tabela 4), comprovando que a seleção para escores visuais trará rápido progresso genético para essas características. No entanto, o mesmo não ocorreu para as estimativas de herdabilidade direta das características aspectos raciais e sacro, o que era previsível, uma vez que na fase da desmama, estas características(Re SAC) ainda não estão bem definidas morfologicamente, o que poderia explicar esta maior contribuição do efeito residual.

Para as características avaliadas por escores visuais de musculosidade e conformação, as estimativas de herdabilidade direta obtidas neste estudo foram semelhantes aos valores encontrados por Cardoso et al. (2001), Jorge Júnior et al. (2004) e Koury Filho (2005), que variaram de 0,11 a 0,34 para musculosidade e 0,10 a 0,33 para conformação, em bovinos Nelore avaliadosà desmama. No entanto, nestes trabalhos citados, a covariância genética aditiva entre os efeitos direto e materno foi considerada nula e a metodologia empregada foi a Máxima Verossimilhança Restrita sob modelo animal linear. É importante ressaltar que o uso da metodologia de modelos lineares pode não ser adequado para características categóricas, por gerar baixas estimativas dos parâmetros genéticos e conclusões 
Tabela 3 - Estatística descritiva das estimativas de herdabilidade das características musculosidade, estrutura física, aspectos raciais, conformação, ônfalo e sacro de bovinos da raça Nelore, em diferentes idades, obtidas a partir de análises bayesianas bicaracterísticas sob modelo animal linear-limiar

\begin{tabular}{|c|c|c|c|c|c|c|}
\hline \multirow[t]{2}{*}{ Estatística descritiva } & \multicolumn{6}{|c|}{ Herdabilidade } \\
\hline & Musculosidade & Estrutura física & Aspectos raciais & Conformação & Ônfalo & Sacro \\
\hline \multicolumn{7}{|l|}{8 meses de idade } \\
\hline Média & 0,32 & 0,60 & 0,22 & 0,34 & 0,57 & 0,08 \\
\hline Moda & 0,31 & 0,60 & 0,21 & 0,33 & 0,79 & 0,06 \\
\hline Mediana & 0,32 & 0,60 & 0,21 & 0,34 & 0,59 & 0,08 \\
\hline Erro de Monte Carlo DP & 0,0014 & 0,0029 & 0,0033 & 0,0024 & 0,0070 & 0,0015 \\
\hline \multicolumn{7}{|l|}{15 meses de idade } \\
\hline Média & 0,38 & 0,14 & 0,21 & 0,07 & 0,36 & 0,46 \\
\hline Moda & 0,38 & 0,14 & 0,22 & 0,07 & 0,31 & 0,46 \\
\hline Mediana & 0,38 & 0,14 & 0,21 & 0,07 & 0,34 & 0,46 \\
\hline Erro de Monte Carlo ${ }^{\text {DP }}$ & 0,0032 & 0,0008 & 0,0037 & 0,0008 & 0,0125 & 0,0066 \\
\hline \multicolumn{7}{|l|}{22 meses de idade } \\
\hline Média & 0,21 & 0,27 & 0,20 & 0,29 & 0,38 & 0,26 \\
\hline Moda & 0,22 & 0,28 & 0,21 & 0,31 & 0,38 & 0,28 \\
\hline Mediana & 0,21 & 0,28 & 0,20 & 0,31 & 0,38 & 0,26 \\
\hline Erro de Monte Carlo ${ }^{\mathrm{DP}}$ & 0,0021 & 0,0018 & 0,0019 & 0,0025 & 0,0020 & 0,0033 \\
\hline \multicolumn{7}{|l|}{ Vacas adultas } \\
\hline Média & 0,01 & 0,07 & 0,18 & 0,11 & 0,36 & 0,23 \\
\hline Moda & 0,01 & 0,06 & 0,17 & 0,09 & 0,37 & 0,23 \\
\hline Mediana & 0,01 & 0,07 & 0,18 & 0,10 & 0,36 & 0,23 \\
\hline Erro de Monte Carlo ${ }^{\mathrm{DP}}$ & 0,0003 & 0,0004 & 0,0024 & 0,0020 & 0,0021 & 0,0039 \\
\hline
\end{tabular}

DP - Desvio-padrão.

equivocadas referentes ao ganho genético destas características quando submetidas à seleção (Van Tassel et al., 1998; Luo et al., 2002; Silva et al., 2003; Marcondes et al., 2005).

As estimativas de herdabilidade do efeito genético aditivo materno foram pequenas para musculosidade, estrutura física e conformação (Tabela 4), o que está de acordo com os relatos de Koury Filho (2005), que estimou a herdabilidade maternal de 0,05 para conformação e 0,06 para musculosidade empregando o modelo linear. Resultados semelhantes em bovinos da raça Nelore foram encontrados por Eler et al. (1996), porém, os dados de escores visuais eram de bovinos Nelore avaliados ao sobreano e esperava-se que a herdabilidade maternal fosse de menor magnitude.

As correlações genéticas entre os efeitos genéticos aditivos diretos e maternos foram de grande magnitude, porém, positivas para musculosidade, conformação e sacro, e negativas paraestrutura física e aspectos raciais. Esses resultados sugerem que os escores visuais para musculosidade, conformação e sacro são fortemente dependentes do efeito materno. No entanto, estes valores positivos de grande magnitude para a associação entre os dois efeitos sugerem mais pesquisas, uma vez que esses valores levariam a grande aumento daherdabilidade total.
Eler et al. (1996) obtiveram estimativas negativas de grande magnitude para covariância genética entre os efeitos direto e materno para musculosidade e conformação. É importante ressaltar que, neste estudo, as estimativas de covariâncias genéticas entre os efeitos genéticos aditivos direto e materno apresentaram dificuldade de convergência da cadeia de Gibbs. Carnier et al. (2000), em análise bicaracterística sob modelo animal de limiar, não conseguiram estimar as correlações genéticas entre os efeitos genéticos aditivos direto e materno. De acordo com Van Tassel et al. (1998), esse fato pode ocorrer em análises de limiar sob modelo animal e gerar estimativas viesadas. No entanto, para que estas correlações genéticas entre os efeitos aditivos direto e materno sejam estimadas com acurácia, é necessário que se tenham dados também das mães e avós dos animais avaliados, o que não ocorreu no conjunto de dados utilizados neste estudo. Robinson (1996) relatou as dificuldades de separar os efeitos genéticosaditivos direto e materno nos modelos estatísticos e a necessidade de se ter uma boa estrutura de dados.

As estimativas de herdabilidade foram baixas para estrutura racial e conformação avaliadas aos 15 meses de idade. Esses resultados podem indicar a interferência de lotes de manejo heterogêneos no momento da avaliação 
Tabela 4 - Médias posteriores das estimativas dos parâmetros genéticos para as características musculosidade, estrutura física, aspectos raciais, conformação e sacro de bovinos da raça Nelore em diferentes idades obtidas a partir de análises bayesianas bicaracterísticas sob modelo animal de limiar

\begin{tabular}{|c|c|c|c|c|c|c|}
\hline \multirow[t]{2}{*}{ Parâmetro genético } & \multicolumn{5}{|c|}{ Características de limiar } & \multirow[b]{2}{*}{ Sacro } \\
\hline & Musculosidade & Estrutura física & Aspectos raciais & Conformação & Ônfalo & \\
\hline \multicolumn{7}{|l|}{8 meses de idade } \\
\hline$\sigma_{\mathrm{d}}^{2}$ & 1,43 & 2,03 & 0,65 & 0,99 & 1,93 & 0,22 \\
\hline$\sigma_{\mathrm{dm}}$ & 0,71 & $-0,54$ & $-0,39$ & 0,11 & - & 0,25 \\
\hline$\sigma_{m}^{2}$ & 0,36 & 0,17 & 0,41 & 0,08 & - & 0,60 \\
\hline$\sigma_{c}^{2}$ & 0,82 & 0,66 & 1,23 & 0,71 & 0,00 & 0,49 \\
\hline $\mathrm{h}^{2}{ }_{\mathrm{d}}^{\mathrm{c}}$ & 0,32 & 0,60 & 0,22 & 0,34 & 0,57 & 0,08 \\
\hline $\mathrm{h}^{2}{ }_{\mathrm{m}}^{\mathrm{a}}$ & 0,07 & 0,05 & 0,14 & 0,03 & - & 0,23 \\
\hline$r_{d m}$ & 0,99 & $-0,94$ & $-0,71$ & 0,42 & - & 0,63 \\
\hline$c^{2+11}$ & 0,19 & 0,20 & 0,41 & 0,24 & 0,00 & 0,19 \\
\hline $\mathrm{e}^{2}$ & 0,25 & 0,31 & 0,35 & 0,35 & 0,43 & 0,42 \\
\hline \multicolumn{7}{|l|}{15 meses de idade } \\
\hline$\sigma^{2}$ & 1,18 & 0,22 & 0,47 & 0,12 & 0,65 & 1,22 \\
\hline$\sigma^{2}{ }^{a}$ & 0,84 & 0,35 & 0,60 & 0,72 & 0,12 & 0,18 \\
\hline$h^{2}$ & 0,38 & 0,14 & 0,21 & 0,07 & 0,36 & 0,46 \\
\hline$c^{2}$ & 0,28 & 0,22 & 0,29 & 0,38 & 0,07 & 0,08 \\
\hline$e^{2}$ & 0,34 & 0,64 & 0,50 & 0,55 & 0,57 & 0,46 \\
\hline \multicolumn{7}{|l|}{22 meses de idade } \\
\hline$\sigma^{2}$ & 0,34 & 0,49 & 0,34 & 0,67 & 0,64 & 0,54 \\
\hline$\sigma^{2}{ }^{2}$ & 0,28 & 0,26 & 0,34 & 0,59 & 0,04 & 0,46 \\
\hline$h^{2}$ & 0,21 & 0,27 & 0,20 & 0,29 & 0,38 & 0,26 \\
\hline$c^{2}$ & 0,17 & 0,15 & 0,20 & 0,26 & 0,03 & 0,23 \\
\hline $\mathrm{e}^{2}$ & 0,62 & 0,58 & 0,60 & 0,45 & 0,59 & 0,51 \\
\hline \multicolumn{7}{|l|}{ Vacas adultas } \\
\hline$\sigma^{2}{ }_{a}$ & 0,01 & 0,07 & 0,27 & 0,12 & 0,58 & 0,31 \\
\hline$\sigma^{2}{ }^{a}$ & 0,00 & 0,00 & 0,20 & 0,00 & 0,00 & 0,00 \\
\hline $\mathrm{h}^{2^{\mathrm{c}}}$ & 0,01 & 0,07 & 0,18 & 0,11 & 0,36 & 0,23 \\
\hline$c^{2}$ & 0,00 & 0,00 & 0,14 & 0,00 & 0,00 & 0,00 \\
\hline$e^{2}$ & 0,99 & 0,93 & 0,68 & 0,89 & 0,64 & 0,77 \\
\hline
\end{tabular}

$\sigma^{2}{ }_{d}=$ variância genética aditiva direta; $\sigma^{2}=$ variância genética aditiva materna; $r_{d m}=$ correlação genética aditiva direta e maternal; $\sigma^{2}=$ variância do efeito de grupo contemporâneo; $\mathrm{h}^{2}{ }_{\mathrm{d}}=$ herdabilidade aditiva direta; $\mathrm{h}^{2}{ }_{\mathrm{m}}=$ herdabilidade aditiva materna; $\mathrm{h}^{2}=$ herdabilidade; $\mathrm{c}^{2}=$ proporção da variância total atribuída ao efeito de grupo contemporâneo; $\mathrm{e}^{2}=$ proporção da variância total devido aos efeitos residuais; $\sigma^{2}{ }_{\mathrm{a}}=$ variância genética aditiva.

visual. Em grandes propriedades, é comum a formação de lotes de manejo no pós-desmame com grandes diferenças de idade entre os animais, principalmentequando não há estação de acasalamentos bem estabelecida. Assim, diferentes épocas de nascimento determinam diferentes épocas de desmama, ocasionando lotes heterogêneos ao sobreano. Porém, a condição primordial para se ter uma boa avaliação visual é a comparação de animais dentro de um mesmo grupo de contemporâneos. De acordo com Koury Filho (2005), quanto menores as diferenças de idade nos lotes apartados para a avaliação visual, mais adequada será a comparação e os resultados tendem a melhores distribuições dentro dos grupos de contemporâneos, além de facilitar o trabalho dos técnicos.

As estimativas de herdabilidade encontradas neste estudo para características morfológicas avaliadas ao sobreano são semelhantes às da literatura. Koury Filho (2001) encontrou estimativas de 0,11 para conformação,
0,11 para precocidade e 0,18 para musculosidade utilizando o método da Máxima Verossimilhança Restrita sob modelo animal linear em dados de bovinos Nelore. Van Melis et al. (2003) estimaram as herdabilidades para conformação, precocidade e musculosidade pelo método $\mathrm{R}$ e encontraram os valores de 0,22;0,21 e 0,22, respectivamente. Koury Filho (2005), trabalhando com dados de bovinos Nelore, estimou a herdabilidade de 0,24 para conformação, 0,32 para precocidade e 0,27 para musculosidade.

Para as características morfológicas avaliadas aos 22 meses de idade, no entanto, as estimativas de herdabilidade foram de média a alta magnitude para todas as características estudadas, indicando que pode ser obtido progresso genético com a seleção individual para essas características. As estimativas de herdabilidade para as características morfológicas avaliadas em vacas adultas foram baixas, tanto para estrutura física como para conformação, e próximas de zero para musculosidade. 
Esses resultados indicam que, para vacas adultas, as avaliações visuais não são indicadas como critérios de seleção e que a seleção deve ocorrer em idades mais jovens. Além disso, os escores para as vacas adultas foram relativos a um padrão considerado ideal para a raça e não em relação ao grupo de contemporâneos, o que pode ter prejudicado a análise genética, em razão da estrutura dos dados.

Os resultados obtidos permitem inferir que os escores visuais, quando criteriosamente aplicados, podem ser utilizados como critérios de seleção para promover ganho genético direto. É importante ressaltar que, para criatórios de bovinos de corte que utilizam a seleção em idades mais jovens, não se justifica a inclusão de características morfológicas aos 22 meses de idade como critérios de seleção e, nesse caso, parece ser mais indicada a inclusão de características morfológicas avaliadas aos 15 meses de idade. No entanto, as características estrutura física e conformação avaliadas aos 15 meses de idade em animais criados em regime de pastejo, podem não apresentar seu total desenvolvimento, daí a diminuição da variabilidade de estrutura física e conformação aos 15 meses. Notou-se também que ônfalo foi a característica morfológica que apresentou maior variabilidade genética (considerando todas as idades) e que aspectos raciais manteve as estimativas de herdabilidade em torno de 0,20 para todas as idades consideradas. Vale ressaltar que, para ônfalo e aspectos raciais, a avaliação visual é realizada com base em notas absolutas e não notas relativas (com base nos grupos de animais contemporâneos), como ocorrem com as demais características (ver explicação em material e métodos).

De modo geral, as estimativas de correlação genética entre as características de musculosidade, estrutura física e conformação foram de magnitude moderada, porém positiva (Tabela 5), indicando que a seleção direta para uma destas características também trará progresso genético às outras, considerando escores visuais avaliados na mesma fase de vida do animal. Esses resultados estão de acordo com os relatados por Cardoso et al. (2004), Jorge Júnior et al. (2004) e Koury Filho (2005), que estimaram correlações genéticas de magnitudes moderadas a altas entre os escores visuais para conformação, precocidade e musculosidade, avaliados em uma mesma fase de vida do animal.

A musculosidade apresentou maior associação genética com conformação, mostrando que ambas as características são influenciadas, em grande parte, pelos mesmos conjuntos de genes. Em relação à estrutura física, observou-se menor associação genética com a musculosidade, no entanto, não há evidências de antagonismo entre estas características. Esses resultados indicam que o desenvolvimento e a distribuição muscular apresentam resposta correlacionada de baixa magnitude com a sustentação do animal. Para estrutura física e conformação, observou-se considerável associação genética entre estas características, exceto aos 8 meses de idade, em que a estimativa foi praticamente nula.

As estimativas de correlação genética entre musculosidade e estrutura física diminuíram à medida que aumentou a classe de idade dos animais avaliados. O contrário ocorreu entre as características estrutura física e conformação, em que as estimativas de correlação genética foram mais altas em idades mais avançadas. Para conformaçãoe musculosidade, as estimativas de correlação genética foram semelhantes em qualquer classe de idade dos animais avaliados. Desta maneira, pode-se inferir que as características de musculosidade, estrutura física e conformação, embora apresentem estimativas altas de herdabilidades ao desmame, podem não estar bem definidas nesta fase de idade, sendo que as diferenças gênicas entre elas podem ser melhor detectadas em idades mais avançadas. Além disso, é importante destacar que os animais avaliados neste estudo foram criados em sistema extensivo, o que pode justificar melhor interpretação das correlações genéticas aos 15 e 22 meses de idade.

Tabela 5 - Estimativas de correlações genéticas (acima da diagonal) e fenotípicas (abaixo da diagonal) entre as características de musculosidade, estrutura física e conformação de bovinos da raça Nelore nas diferentes idades $(8,15$ e 22 meses de idade), obtidas a partir de análises bicaracterísticas sob modelo animal de limiar

\begin{tabular}{|c|c|c|c|c|c|c|c|c|c|}
\hline & $\mathrm{M}_{8}$ & $\mathrm{E}_{8}$ & $\mathrm{C}_{8}$ & $\mathrm{M}_{15}$ & $\mathrm{E}_{15}$ & $\mathrm{C}_{15}$ & $\mathrm{M}_{22}$ & $\mathrm{E}_{22}$ & $\mathrm{C}_{22}$ \\
\hline $\mathrm{M}_{8}$ & 1 & 0,40 & 0,61 & 0,30 & 0,16 & 0,21 & 0,05 & $-0,09$ & 0,01 \\
\hline $\mathrm{E}_{8}{ }^{\circ}$ & 0,58 & 1 & $-0,07$ & 0,16 & 0,30 & 0,13 & 0,08 & 0,04 & 0,14 \\
\hline $\mathrm{C}_{8}$ & 0,46 & 0,60 & 1 & 0,22 & 0,13 & 0,10 & $-0,03$ & $-0,07$ & 0,04 \\
\hline $\mathrm{M}_{15}$ & 0,18 & 0,13 & 0,08 & 1 & 0,23 & 0,47 & 0,59 & 0,31 & 0,47 \\
\hline $\mathrm{E}_{15}$ & 0,22 & 0,12 & 0,13 & 0,39 & 1 & 0,38 & 0,27 & 0,50 & 0,33 \\
\hline $\mathrm{C}_{15}$ & 0,14 & 0,03 & 0,13 & 0,49 & 0,42 & 1 & 0,61 & 0,43 & 0,54 \\
\hline $\mathrm{M}_{22}$ & 0,13 & 0,01 & 0,00 & 0,27 & 0,18 & 0,24 & 1 & 0,18 & 0,75 \\
\hline $\mathrm{E}_{22}$ & 0,12 & 0,01 & 0,10 & 0,18 & 0,25 & 0,20 & 0,49 & 1 & 0,56 \\
\hline $\mathrm{C}_{22}$ & 0,10 & 0,04 & 0,11 & 0,25 & 0,20 & 0,22 & 0,64 & 0,51 & 1 \\
\hline
\end{tabular}


Quando as correlações genéticas entre a mesma característica morfológica foram realizadas considerando as diferentes idades, as estimativas entre as idades de 8 e 22 meses foram praticamente nulas entre musculosidade, estrutura física e conformação. Entretanto, quando as características foram avaliadas em idades próximas, por exemplo, 8 e 15 meses de idade ou 15 e 22 meses de idade, as correlações genéticas foram moderadas para todas as características morfológicas estudadas, exceto entre conformação aos 8 e 15 meses de idade. Esses resultados parecem indicar a necessidade de se utilizar informações de escores visuais em, pelo menos, duas fases de vida do animal como critérios de seleção. No entanto, é importante ressaltar que, para as análises que consideraram as características morfológicas aos 8 e 22 meses de idade, somente $15 \%$ dos animais avaliados possuíam dados para ambas características, ou seja, as estimativas de correlações genéticas foram obtidas por meio das informações da matriz de parentesco.

Resultados semelhantes foram obtidos por Koury Filho (2005), que encontrou estimativas altas para as correlações genéticas entre conformação, precocidade e musculosidade na desmama e ao sobreano em bovinos da raça Nelore. Estimativas mais altas foram obtidas por Cardoso et al. (2004) para conformação, precocidade e musculosidade na desmama e sobreano para animais da raça Angus. Os mesmos autores sugeriram a avaliação por escores apenas no momento da desmama, com intuito de evitar perdas de informações por pré-seleção. No entanto, Koury Filho (2005) considera precipitada a decisão de um único momento para se avaliar os animais, pois, se a avaliação na desmama é muito interessante por não haver pré-seleção, ao sobreano as características morfológicas expressam melhor o potencial genético direto do indivíduo. De acordo com os resultados obtidos, as características morfológicas avaliadas ao desmame e ao sobreano podem ser recomendadas como critérios de seleção.

\section{Conclusões}

As características morfológicas de musculosidade, estrutura física, aspectos raciais, conformação, ônfalo e osso sacro apresentaram possibilidade de resposta rápida à seleção direta e, portanto, devem ser incorporadas em programas de melhoramento genético como critérios de seleção. Com base nas baixas estimativas de correlações genéticas entre a desmama e as demais idades, recomenda-se avaliar estas características morfológicas tanto nadesmama quanto ao sobreano com fim de seleção.

\section{Agradecimentos}

À ANCP, ao Departamento de Genética e Melhoramento da UNESP/Jaboticabal, à Escola de Veterinária da UFG e à Embrapa Arroz e Feijão, pelo apoio técnico-científico, e ao $\mathrm{CNPq}$, pelo suporte financeiro.

\section{Literatura Citada}

CARDOSO, F.F.; CARDELliNO, R.A.; CAMPOS, L.T. Fatores ambientais sobre escores de avaliação visual a desmama em bezerros Angus criados no Rio Grande do Sul. Revista Brasileira de Zootecnia, v.30, n.2, p.318-325, 2001.

CARDOSO, F.F.; CARDELlinO, R.A.; CAMPOS, L.T. Componentes de (co)variâncias e parâmetros genéticos de caracteres pós-desmama em bovinos da raça Angus. Revista Brasileira de Zootecnia, v.33, n.2, p.313-319, 2004.

CARNIER, P.; ALBERA, A.; DAL ZOTTO, R. et al. Genetic parameters for direct and maternal calving ability over parities in Piedmontese cattle. Journal Animal Science, v.78, p.2532-2539, 2000 .

ELER, J.P.; FERRAZ, J.B.S.; SILVA, P.R. Parâmetros genéticos para peso, avaliação visual e circunferência escrotal na raça Nelore, estimados por modelo animal. Arquivo Brasileiro de Medicina Veterinária e Zootecnia, v.48, n.2, p.203-213, 1996.

GIANOLA, D.; FOULLEY, J.L. Sire evaluation for orderer categorical data with a threshold model. Genetic Selection Evolution, v.15, p.201-224, 1983.

GIANOLA, D.; SORENSEN, D. Likelihood, bayesian, and MCMC methods in quantitative genetics. Statistics for Biology and Health. s.l: Springer, 2002. 740p.

JORGE JR., J.; DIAS, L.T.; ALBUQUERQUE, L.G. Fatores de correção de escores visuais de conformação, precocidade e musculatura, à desmama, para idade da vaca ao parto, data Juliana de nascimento e idade à desmama em bovinos da raça Nelore. Revista Brasileira de Zootecnia, v.33, n.6, p.20442053, 2004.

KOURY FILHO, W.Análise genética de escores visuais e suas respectivas relações com desempenho ponderal na raça Nelore. 2001. 71f. Dissertação (Mestrado em Zootecnia) Universidade Estadual Paulista, 2001, Jaboticabal.

KOURY FILHO, W. Escores visuais e suas relações com características de crescimento em bovinos de corte. Universidade Estadual Paulista, 2005. 80f. Tese (Doutorado em Zootecnia) - Universidade Estadual Paulista, 2005, Jaboticabal.

LÔBO, R.B. Programa de melhoramento genético da raça Nelore. Ribeirão Preto: FINEP, 1996. 100p.

LÔBO, R.B.; BEZERRA, L.A.F.; OLIVEIRA, H.N. et al.Avaliação genética de touros e matrizes da raça Nelore: sumário 2006. Ribeirão Preto: ANCP, 2006. 124p.

LUO, M.F.; BOETTCHER, P.J.; SCHAEFFER, L.R. et al. Estimation of genetic parameters of calving ease in first and second parities of Canadian Holsteins using Bayesian methods. Livestock Production Science, v.74, p.175-184, 2002.

MARCONDES, C.R.; PANETO, J.C.C.; SILVA, J.A.V. et al. Comparação entre análises para permanência no rebanho de vacas Nelore utilizando modelo linear e modelo de limiar. Arquivo Brasileiro de Medicina Veterinária e Zootecnia, v.57, n.2, p.234-240, 2005.

MEYER, K. Variance components due to direct and maternal effects for growth traits of Australian beef cattle. Livestock Production Science, v.1, p.179, 1992.

MORENO, C.; SORENSEN, D.; GARCIA-CORTÉS, L.A. et al. On biased inferences about variance components in the binary 
threshold model. Genetic Selection Evolution, v.29, p.145-160, 1997.

MWANSA, P.B.; CREWS, D.H.J.; WILTON, J.W. et al. Multiple trait selection for maternal productivity in beef cattle. Journal Animal Breeding Genetics, v.119, p.391-399, 2002.

RAMIREZ VALVERDE, R.; MIZTAL, I.; BERTRAND, J.K. Comparison of threshold vs linear and animal vs sire models for predicting direct and maternal genetic effects on calving difficulty in beef cattle. Journal of Animal Science, v.79, p.333-338, 2001

ROBINSON, D.L. Estimation and interpretation of direct and maternal genetic parameter for weights of Australian Angus cattle. Livestock Production Science, v.45, p.1-11, 1996.

STATISTICAL ANALYSIS SYSTEM - SAS. SAS OnlineDoc ${ }^{\circledR}$ 9.1.3. Cary: SAS Institute, 2004. (CD-ROM).

SILVA, J.A.V.; ELER, J.P.; FERRAZ, J.B.S. et al. Análise genética da habilidade de permanência em fêmeas da raça Nelore. Revista Brasileira de Zootecnia, v.32, n.3, p.598-604, 2003.

SILVA, J.A.V.; DIAS, L.T.; ALBUQUERQUE, L.G. Estudo genético da precocidade sexual de novilhas em um rebanho
Nelore. Revista Brasileira de Zootecnia, v.34, n.5, p.1568-1572, 2005.

SORENSEN, D.A.; ANDERSEN, S.; GIANOLA, D. et al. Bayesian inference in threshold models using Gibbs sampling. Genetic Selection Evolution, v.27, p.229-249, 1995.

Van MELIS, M.H.; ELER, J.P.; SILVA, J.A.V. et al. Estimação de parâmetros genéticos em bovinos de corte utilizando os métodos de Máxima Verossimilhança Restrita e R. Revista Brasileira de Zootecnia, v.32, n.6, p.1624-1632, 2003.

Van TASSELL, C.P.; Van VLECK, L.D.; GREGORY, K. E. Bayesian analysis of twinning and ovulation rates using a multiple-trait threshold model and Gibbs sampling. Journal of Animal Science, v.76, p.2048-2061, 1998.

Van KAAM, J.B.C.H.M. Gibanal 2.9: analyzing program for Markov Chain Monte Carlo Sequences. Wageningen: Wageningen Agricultural University, 1998. (CD-ROM).

VARONA, L.; MIZTAL, I.; BERTRAND, J.K. Threshold-linear versus linear-linear analysis of birth weight and calving ease using an animal model: I. Variance component estimation. Journal of Animal Science, v.77, p.1994-2002, 1999. 